\title{
Daily chocolate consumption is inversely associated with insulin resistance and liver enzymes in the Observation of Cardiovascular Risk Factors in Luxembourg study
}

\author{
Ala'a Alkerwi ${ }^{1 *}$, Nicolas Sauvageot ${ }^{1}$, Georgina E. Crichton ${ }^{1,2}$, Merrill F. Elias ${ }^{3,4}$ and Saverio Stranges ${ }^{1,5}$ \\ ${ }^{1}$ Luxembourg Institute of Health (LIH) (formerly CRP-Santé), Epidemiology and Public Health Research Unit, Strassen, \\ L-1445, Grand-Duchy of Luxembourg \\ ${ }^{2}$ Nutritional Physiology Research Centre, University of South Australia, Adelaide 5001, Australia \\ ${ }^{3}$ Department of Psychology, University of Maine, Orono, ME 04469, USA \\ ${ }^{4}$ Graduate School of Biomedical Science and Engineering, University of Maine, Orono, ME O4469, USA \\ ${ }^{5}$ Division of Health Sciences, University of Warwick Medical School, Coventry CV4 7AL, UK
}

(Submitted 16 November 2015 - Final revision received 13 January 2016 - Accepted 1 February 2016 - First published online 17 March 2016$)$

\section{Abstract}

This study examined the association of chocolate consumption with insulin resistance and serum liver enzymes in a national sample of adults in Luxembourg. A random sample of 1153 individuals, aged 18-69 years, was recruited to participate in the cross-sectional Observation of Cardiovascular Risk Factors in Luxembourg study. Chocolate consumption (g/d) was obtained from a semi-quantitative FFQ. Blood glucose and insulin levels were used for the homoeostasis model assessment of insulin resistance (HOMA-IR). Hepatic biomarkers such as serum $\gamma$-glutamyl-transpeptidase $(\gamma$-GT), serum aspartate transaminase and serum alanine transaminase (ALT) (mg/l) were assessed using standard laboratory assays. Chocolate consumers $(81.8 \%)$ were more likely to be younger, physically active, affluent people with higher education levels and fewer chronic co-morbidities. After excluding subjects taking antidiabetic medications, higher chocolate consumption was associated with lower HOMA-IR $(\beta=-0 \cdot 16, P=0 \cdot 004)$, serum insulin levels $(\beta=-0 \cdot 16, P=0 \cdot 003)$ and $\gamma$-GT $(\beta=-0 \cdot 12, P=0 \cdot 009)$ and ALT $(\beta=-0.09, P=0.004)$, after adjustment for age, sex, education, lifestyle and dietary confounding factors, including intakes of fruits and vegetables, alcohol, polyphenol-rich coffee and tea. This study reports an independent inverse relationship between daily chocolate consumption and levels of insulin, HOMA-IR and liver enzymes in adults, suggesting that chocolate consumption may improve liver enzymes and protect against insulin resistance, a well-established risk factor for cardiometabolic disorders. Further observational prospective research and well-designed randomised-controlled studies are needed to confirm this cross-sectional relationship and to comprehend the role and mechanisms that different types of chocolate may play in insulin resistance and cardiometabolic disorders.

Key words: Insulin resistance: Liver enzymes: Chocolate consumption

Atherosclerotic CVD is the leading killer in the adult population of Western societies ${ }^{(1)}$. Obesity, diabetes and insulin resistance are metabolic disorders and well-known risk factors for $\mathrm{CVD}^{(2-4)}$. Hyperinsulinaemia has been associated with an increased cardiovascular risk in non-diabetic subjects, and has been related to a number of other conditions such as hypertension, dyslipidaemia and central body fat distribution ${ }^{(5)}$. These pathologies are escalating worldwide and constitute an important concern to public health authorities.

Cocoa research has received much attention over recent years. Chocolate may have beneficial cardiometabolic effects, possibly due to cocoa polyphenols. Cocoa beans and its derivatives contain different types of physiologically active compounds including, among others, polyphenols and their flavonoids subclasses ${ }^{(6,7)}$. Cocoa, especially dark chocolate, represents a noteworthy source of flavonoids as it contains considerably higher concentrations of flavonoids per serving than tea, apple and red wine ${ }^{(8,9)}$, contributing to its higher antioxidant capacity and, presumably, to its beneficial health effects $^{(10)}$. Epidemiological data suggest that flavonoid intake from different sources may reduce the risk of $\mathrm{CHD}^{(11-13)}$ and stroke $^{(14)}$ and is inversely associated with coronary mortality ${ }^{(15)}$.

A recent experimental study on obese mice reported that cocoa supplementation attenuated insulin resistance, as indicated by improved homoeostasis model assessment of insulin resistance (HOMA-IR), and reduced the severity of

Abbreviations: FPG, fasting plasma glucose; HOMA-IR, homoeostasis model assessment of insulin resistance; ORISCAV-LUX, Observation of Cardiovascular Risk Factors in Luxembourg.

* Corresponding author: A. Alkerwi, fax +352 26970 719, email alaa.alkerwi@lih.lu 
obesity-related fatty liver disease, a condition implicated in diabetes risk ${ }^{(16)}$. In line with these findings, a small, short-term, cross-over study has reported that cocoa ingestion improved insulin sensitivity in healthy subjects ${ }^{(17)}$. Another small randomised trial showed increased insulin sensitivity in glucoseintolerant, hypertensive subjects after $15 \mathrm{~d}$ of consuming high-polyphenol dark chocolate ${ }^{(18)}$. Thus far, evidence regarding a potential role of chocolate intake in insulin resistance and liver function from human epidemiological studies is lacking.

In the present study, we hypothesised that chocolate consumption may have a beneficial effect on insulin sensitivity and liver enzymes. Therefore, we examined whether daily chocolate consumption may be associated with insulin resistance and serum insulin and liver enzyme levels in a national sample of adults in Luxembourg, taking into account potential confounding lifestyle and dietary factors, including the simultaneous consumption of polyphenol-rich tea and coffee beverages.

\section{Methods}

\section{Study design and participants}

Analyses were based on data from the Observation of Cardiovascular Risk Factors in Luxembourg (ORISCAV-LUX) survey, a nationwide population-based, cross-sectional study of the adult population in Luxembourg. More details of the study design, sample selection and data collection have been previously reported $^{(19,20)}$. In brief, a stratified random sample of 1432 participants, aged 18-69 years, was recruited between November 2007 and January 2009. Trained research staff provided the participants with detailed instructions on how to complete the self-administered $\mathrm{FFQ}^{(21,22)}$, assisted them in completing questions on dietary information and then checked the completeness and accuracy of responses. After data cleaning, particularly for poorly completed dietary data, 1352 participants were available for the present study. Analyses were carried out with a set of 1153 subjects after eliminating those who reported dieting during the survey period ( $n$ 199).

The ORISCAV-LUX study was conducted according to the guidelines laid down in the Declaration of Helsinki. All procedures involving human subjects were approved by the National Research Ethics Committee (N 200609/03) and the National Commission for Private Data Protection. Written informed consent was obtained from all subjects.

\section{Insulin resistance and liver biomarkers (dependent variables)}

Standard laboratory assays were used to assess several biomarkers including fasting plasma glucose (FPG, mg/dl), serum insulin $(\mu \mathrm{g} / \mathrm{l})$, glycated $\mathrm{Hb}(\mathrm{HbA} 1 \mathrm{c})$ in percentage values and hepatic biomarkers (serum $\gamma$-glutamyl-transpeptidase $(\gamma$-GT $)$, serum aspartate transaminase and serum alanine transaminase (ALT) (mg/l)). Similar to most epidemiological studies, the HOMA-IR was used as a feasible surrogate approach to assess insulin sensitivity ${ }^{(23)}$, and was calculated as follows: HOMA-IR $=$ (fasting glucose $\times$ fasting insulin/22.5). Lower scores indicate higher insulin sensitivity.

\section{Chocolate consumption (exposure-independent variables)}

A semi-quantitative, 134-item FFQ was completed by the participants, including questions on habitual daily consumption of chocolate during the previous 3 months. The participants reported their frequency of consumption from six response options, ranging from 'rarely or never' (i.e. less than once per month) to 'two or more times per d'. They also selected the serving size of chocolate based on a photographic manual ${ }^{(19)}$ provided as a reference (e.g. one portion is equal to one pre-packaged bar of chocolate). Frequency of consumption (times per $\mathrm{d}$ ) $\times$ amount of chocolate consumed $(\mathrm{g})$ represented daily chocolate consumption $(\mathrm{g} / \mathrm{d})$. To facilitate data interpretation, daily chocolate consumption was then multiplied by 100 to convert it to $100 \mathrm{~g} / \mathrm{d}$.

\section{Covariates}

Demographic and socio-economic data were obtained from a self-administered questionnaire (age, sex, education). Education was grouped into primary, secondary or tertiary levels. Detailed data regarding cigarette smoking were obtained from the health questionnaire, and each participant was classified as current smoker or non-smoker. Self-reported time per week spent engaging in physical activity was assessed using the International Physical Activity Questionnaire (IPAQ), and was used to classify the participants into 'inactive', 'moderately active' or 'active', based on scoring criteria of the IPAQ's Research Committee.

BMI was calculated as weight $(\mathrm{kg})$ divided by the square of height $\left(\mathrm{m}^{2}\right)$, measured according to standard operating procedures using a digital column scale (Seca 701; Seca). Waist circumference (WC, cm) was measured at the level midway between the twelfth rib and the uppermost lateral border of the iliac crest during normal expiration. Standard laboratory assays were used to assess lipid biomarkers: TAG ( $\mathrm{mg} / \mathrm{dl})$, HDL-cholesterol $(\mathrm{mg} / \mathrm{dl})$, LDL-cholesterol $(\mathrm{mg} / \mathrm{dl})$ and total cholesterol $(\mathrm{mg} / \mathrm{dl})$. Blood pressure (BP) was measured using an Omron MX3 Plus automated oscillometric blood pressure monitor (O-HEM-742-E). Measurements were taken at least three times with the participants in a seated position and with a minimum interval of $5 \mathrm{~min}$ between each measurement. The average of the last two readings was used in the analysis. All these variables were measured according to ORISCAV-LUX standardised protocols and have been described in detail in previous publications ${ }^{(19,24,25)}$.

Total daily energy intakes $(\mathrm{kJ} / \mathrm{d}(\mathrm{kcal} / \mathrm{d}))$, energy from total carbohydrates, sugars and added sugar, from total fat and SFA (all as \%E) as well as from alcohol $(\mathrm{ml} / \mathrm{d})$, fruit and vegetable consumptions $(\mathrm{g} / \mathrm{d})$ were obtained from the FFQ. These variables have been described in detail elsewhere ${ }^{(25)}$.

\section{Statistical analysis}

To describe chocolate consumption among the studied population, participants were classified into two groups: non-consumers and consumers. According to the type of variable (continuous or categorical), the Wilcoxon's and $\chi^{2}$ tests 
were used to compare demographic, lifestyle and cardiometabolic characteristics across the groups according to daily chocolate consumption. For categorical variables, values were expressed as numbers and percentages, and for continuous variables values were expressed as mean values and standard deviations.

After exclusion of subjects taking hypoglycaemic medication (twenty-five subjects; $2 \cdot 17 \%$ ), linear regression analyses were performed to assess the association between chocolate consumption and FPG, HbA1c, HOMA-IR, insulin and liver enzymes. The coefficient of regression $(b)$ was computed to predict how much a $100 \mathrm{~g}$ increase in chocolate consumption may increase or decrease each outcome variable. Three models with progressive adjustment were used:

(1) Basic covariate set (model I): adjusted for age sex, and education level (primary, secondary or tertiary).

(2) Full covariate set (model II): basic + further adjustment for smoking status (smoker or non-smoker), physical activity (metabolic equivalent-min/week), fruit and vegetable intake $(\mathrm{g} / \mathrm{d})$, total daily energy intake $(\mathrm{kJ} / \mathrm{d}(\mathrm{kcal} / \mathrm{d}))$ and alcohol consumption $(\mathrm{ml} / \mathrm{d})$.

(3) Extended covariate set (model III): full covariate set+ further adjustment for coffee and tea consumption.

Potential confounding factors were identified and included in the models on the basis of two criteria: (1) had to show a statistically significant association $(P<0.05)$ with both chocolate consumption and any hepatic biomarker and (2) had to be theoretically or clinically relevant. Although BMI met the first criterion to be considered as a confounding variable, we decided not to include it in the multivariable models to avoid overadjustment bias, defined as control for an intermediate variable (or a proxy for an intermediate variable) on a causal path from exposure to outcome ${ }^{(26)}$. BMI is likely to act as a mediator (or a proxy for another unmeasured intermediate variable), and thus intervene in the causal pathway of the chocolate-biomarkers relationships.

Plausible interactions between chocolate consumption and BMI were tested and found to be not significant. Several sensitivity analyses were performed to confirm the robustness of the presented findings. Results were considered significant at the $5 \%$ critical level $(P>0.05$, two-sided). All statistical analyses were performed using SAS version 9.4

\section{Results \\ Chocolate consumption}

More than $80 \%$ of the participants reported chocolate consumption, with an average daily consumption of $24.8 \mathrm{~g} / \mathrm{d}$ (minimum 0.66; maximum 500.7) and median intake of $10.7 \mathrm{~g} / \mathrm{d}$ (lower quintile $=5 \cdot 4$; upper quintile $=28 \cdot 6$ )

\section{Participant characteristics according to daily chocolate consumption}

Globally, daily chocolate consumption varied significantly according to demographic, lifestyle and health factors of the
ORISCAV-LUX participants (all $P<0.05$ ), except for sex $(P=0 \cdot 7)$ and tobacco consumption $(P=0 \cdot 11)$. Non-consumers were considerably more likely to have a lower education level, to be living below the poverty threshold and be physically inactive compared with chocolate consumers. Those who consumed chocolate were significantly younger (43.1 v. 48.3 years; $P<0.0001)$ Cardiometabolic co-morbidities (obesity, diabetes and hypertension) were less frequent among chocolate consumers than non-consumers (Table 1).

Table 2 describes dietary and cardiometabolic variables according to daily chocolate consumption. Chocolate consumers had significantly higher intakes of total energy $(P<0.0001)$ and total fats including SFA $(P<0.0001)$, but lower intakes of alcohol $(P=0.032)$, compared with their counterparts. Glycaemic parameters including FPG, HbA1c and HOMA-IR as well as BMI, WC and total cholesterol levels were significantly higher among non-consumers

\section{Association of chocolate consumptions with insulin resistance and liver biomarkers}

Table 3 shows the multivariable regression analysis, relating chocolate consumption $(100 \mathrm{~g} / \mathrm{d})$ to insulin resistance and hepatic markers. A tendency for an inverse relationship between all liver biomarkers and chocolate consumption was observed, indicated by negative regression coefficients $(b)$ for all the models.

Daily chocolate consumption was significantly associated with lower HOMA-IR $(P=0.004)$, serum insulin levels $(P=0.003)$ and liver enzymes including $\gamma$-GT $(P=0.009)$ and ALT $(P=0.004)$, after adjustment for age, sex, education, lifestyle (smoking status and physical activity) and dietary intake of fruits and vegetables, alcohol, coffee and tea (model III). Daily consumption of $100 \mathrm{mg}$ of chocolate was associated with a reduction of HOMA-IR by $0 \cdot 16$, of serum insulin levels by $0 \cdot 16 \mu \mathrm{g} / \mathrm{l}$ and of liver enzymes $(\gamma$-GT, ALT) by $>0 \cdot 10 \mathrm{mg} / \mathrm{l}$. No statistically significant associations of FPG or HbA1c with chocolate consumption were detected, despite the overall negative regression coefficients.

\section{Discussion}

To our knowledge, this is the first cross-sectional, populationbased study among apparently healthy adults to demonstrate an inverse association between daily chocolate consumption and insulin resistance, serum insulin and liver enzymes, independent of age, sex and socio-economic, lifestyle and dietary factors, including the simultaneous consumption of polyphenolrich tea and coffee beverages. Daily consumption of $100 \mathrm{mg}$ of chocolate was associated with lower HOMA-IR (by 0.16) and serum insulin (by $0 \cdot 16 \mu \mathrm{g} / \mathrm{l}$ ). Likewise, liver enzyme levels were reduced by about $0 \cdot 10 \mathrm{mg} / \mathrm{l}$.

Research into the health effects of cocoa has received much attention over the past few years. Several systematic reviews and meta-analyses of existing studies have generally agreed on a potential beneficial association between chocolate consumption and a lower risk for cardiometabolic disorders ${ }^{(6,30,31)}$. 
Table 1. Participant characteristics according to daily chocolate consumption (g/d), Observation of Cardiovascular Risk Factors in Luxembourg 2007-2008*

(Numbers and percentages; $n 1153$ subjects)

\begin{tabular}{|c|c|c|c|c|c|}
\hline \multirow[b]{3}{*}{ Characteristics } & \multicolumn{5}{|c|}{ Daily chocolate consumption (g/d) } \\
\hline & \multicolumn{2}{|c|}{ Non-consumers } & \multicolumn{2}{|c|}{ Consumers } & \multirow[b]{2}{*}{$P+$} \\
\hline & $n$ & $\%$ & $n$ & $\%$ & \\
\hline Participants & 210 & $18 \cdot 2$ & 943 & $81 \cdot 8$ & \\
\hline Sex & & & & & 0.7 \\
\hline Men & 103 & $49 \cdot 1$ & 476 & $50 \cdot 5$ & \\
\hline Women & 107 & 50.9 & 467 & 49.5 & \\
\hline Education level & & & & & $<0.0001$ \\
\hline Primary & 87 & 42.03 & 216 & $23 \cdot 15$ & \\
\hline Secondary & 88 & $42 \cdot 5$ & 444 & $47 \cdot 6$ & \\
\hline Tertiary & 32 & 15.5 & 273 & $29 \cdot 3$ & \\
\hline \multicolumn{6}{|l|}{ Income } \\
\hline Below poverty level & 53 & 28.5 & 158 & $19 \cdot 4$ & 0.006 \\
\hline Current smokers & 54 & $25 \cdot 7$ & 195 & $20 \cdot 7$ & 0.11 \\
\hline Physical activity & & & & & 0.049 \\
\hline Inactive & 45 & $22 \cdot 4$ & 142 & $15 \cdot 7$ & \\
\hline Moderately active & 47 & 23.4 & 259 & $28 \cdot 7$ & \\
\hline Active & 109 & 54.2 & 501 & 55.5 & \\
\hline Obesity & 58 & $27 \cdot 8$ & 173 & $18 \cdot 3$ & 0.002 \\
\hline Hypertension§ & 103 & 49.1 & 325 & 34.5 & $<0.0001$ \\
\hline Diabetes (\%)\| & 15 & $7 \cdot 3$ & 28 & 3.04 & 0.0041 \\
\hline Dyslipidaemiaף & 169 & 82.04 & 671 & $72 \cdot 1$ & 0.23 \\
\hline
\end{tabular}

* Difference in the number of cases is related to missing values for several variables. Physical activity was assessed via scoring criteria of the International Physical Activity Questionnaire.

$\dagger P$ values for testing the differences among demographic and lifestyle characteristic variables across two groups of chocolate consumption by using $\chi^{2}$ test. $P>0.05$ considered significant.

$\ddagger$ Obesity defined as $\mathrm{BMI} \geq 30 \mathrm{~kg} / \mathrm{m}^{2}$.

$\S$ Hypertension defined as systolic blood pressure $\geq 140 \mathrm{mmHg}$ and/or diastolic blood pressure $\geq 90 \mathrm{mmHg}$ and/or taking antihypertensive medications ${ }^{(27)}$.

|| Diabetes defined as taking antidiabetic medications and/or having fasting plasma glucose $\geq 126 \mathrm{mg} / \mathrm{dl}(\geq 7 \mathrm{mmol} / /)^{(28)}$

II Dyslipidaemia was defined as having at least one of the following anomalies: total cholesterol $\geq 190 \mathrm{mg} / \mathrm{dl}$ ( $\geq 4.9 \mathrm{mmol} / \mathrm{l})$, TAG $\geq 150 \mathrm{mg} / \mathrm{dl}$ ( $\geq 1.7 \mathrm{mmol} / \mathrm{l})$, LDL-cholesterol $\geq 115 \mathrm{mg} / \mathrm{dl}$ ( $\geq 3.0 \mathrm{mmol} / \mathrm{l}), \mathrm{HDL}$-cholesterol $<40 \mathrm{mg} / \mathrm{dl}$ for men and $<46 \mathrm{mg} / \mathrm{dl}$ for women ${ }^{(29)}(<1.0 \mathrm{mmol} / \mathrm{l}$ for men and $<1.2 \mathrm{mmol} / \mathrm{l}$ for women) and/or taking hypolipid medications.

In ORISCAV-LUX participants, there was no sex-specific difference in chocolate consumption. A higher number of non-consumers were living below the poverty threshold, had lower education levels and were more physically inactive compared with regular chocolate consumers.

Chocolate is rich in sugar and fat, contributing to the assumption that frequent consumption may boost obesity. In contrast to traditional beliefs, the present study showed that adiposity measures (BMI, WC), glycaemic parameters (FPG, HbAc1 and HOMA-IR) and $\gamma$-GT were significantly lower among chocolate consumers. In addition, obesity and other cardiometabolic-related pathologies such as hypertension and diabetes were significantly lower among daily chocolate consumers. These findings are consistent with an American study focused on candy consumption ${ }^{(32)}$. Therefore, it is also possible that chocolate consumption may represent an overall marker for a cluster of favourable socio-demographic profiles, healthier lifestyle behaviours and better health status. This could explain, at least in part, the observed inverse associations with insulin and liver biomarkers.

In the present study, daily chocolate consumers also had diets higher in energy, with a greater contribution from total fat and SFA. The lipid content of chocolate is relatively high; however, one-third of the lipid in cocoa butter is composed of the fat stearic acid, which exerts a neutral cholesterolaemic response in humans ${ }^{(33)}$. In addition, further adjustment for SFA did not change the findings (data not shown).
Our findings are in line with two small-scale interventional trials. The first study demonstrated that chocolate (cocoa powder) may have specific insulinotropic effects, irrespective of the food source or the overall macronutrient composition of the food $^{(34)}$. The second one, a cross-over trial, showed that consumption of flavanols-rich dark chocolate reduced insulin resistance, as measured by three methods: HOMA, quantitative insulin sensitivity check index and insulin sensitivity index ${ }^{(35)}$. Recently, data from a longitudinal study revealed that daily chocolate intake was associated with reduced levels of liver enzymes in HIV-hepatitis C virus co-infected patients ${ }^{(36)}$.

Cocoa and its derivatives contain a specific class of polyphenols named flavanols, which are recognised as having important anti-inflammatory properties, possibly reducing the risk of $\mathrm{CHD}$, cancer and other inflammatory-related diseases $^{(11,37)}$. Cocoa contains more phenolic antioxidants than most foods. Antioxidant effects of cocoa may directly influence insulin resistance and, in turn, reduce diabetes risk ${ }^{(38)}$. An additional potential mechanism through which cocoa might exert its benefits on insulin sensitivity is dependent, at least in part, on insulin-mediated nitric oxide (NO) release ${ }^{(39)}$. In fact, flavanols may stimulate production of $\mathrm{NO}$ from vascular endothelium and mimic metabolic actions of insulin by using signalling pathways partially overlapping with those regulating vasodilator actions of insulin ${ }^{(40)}$. Thus, flavanols and related polyphenolic antioxidants may counteract insulin resistance by increasing NO bioavailability ${ }^{(7,35)}$. The observed association 
Table 2. Dietary and cardiometabolic variables according to daily chocolate consumption ( $\mathrm{g} / \mathrm{d})$, Observation of Cardiovascular Risk Factors in Luxembourg 2007-2008

(Mean values and standard deviations; $n 1153$ subjects)

\begin{tabular}{|c|c|c|c|c|c|}
\hline \multirow[b]{3}{*}{ Variables } & \multicolumn{5}{|c|}{ Daily chocolate consumption $(\mathrm{g} / \mathrm{d})$} \\
\hline & \multicolumn{2}{|c|}{ Non-consumers } & \multicolumn{2}{|c|}{ Consumers } & \multirow[b]{2}{*}{$P^{*}$} \\
\hline & Mean & SD & Mean & SD & \\
\hline Age (years) $\dagger$ & $48 \cdot 3$ & $12 \cdot 8$ & $43 \cdot 1$ & $12 \cdot 9$ & $<0.0001$ \\
\hline \multicolumn{6}{|l|}{ Dietary variables } \\
\hline Alcohol intake $(\mathrm{ml} / \mathrm{d})$ & 177.5 & 383.4 & $149 \cdot 3$ & 226 & 0.032 \\
\hline Fruit and vegetable intake $(\mathrm{g} / \mathrm{d})$ & 569.96 & 435.6 & 533.7 & $379 \cdot 1$ & 0.39 \\
\hline Total energy intake $(\mathrm{kJ} / \mathrm{d})$ & 8950.8 & 3632.5 & 10479.2 & $127 \cdot 6$ & $<0.0001$ \\
\hline Total energy intake $(\mathrm{kcal} / \mathrm{d})$ & $2139 \cdot 3$ & $868 \cdot 2$ & 2504.6 & 30.5 & $<0.0001$ \\
\hline Total carbohydrate (\%E) & 43.5 & 8.6 & $42 \cdot 4$ & 7.0 & 0.11 \\
\hline Total sugar & $20 \cdot 6$ & 8.3 & 20.7 & 6.5 & 0.20 \\
\hline Added sugar (\%E) & $5 \cdot 3$ & 4.4 & 7.5 & $4 \cdot 1$ & $<0.0001$ \\
\hline Total fat $(\% \mathrm{E})$ & $36 \cdot 7$ & 8.0 & 38.7 & $6 \cdot 7$ & 0.0001 \\
\hline SFA (\%E) & $12 \cdot 1$ & 3.04 & 13.7 & 2.74 & $<0.0001$ \\
\hline \multicolumn{6}{|l|}{ Cardiometabolic variables } \\
\hline FPG $(\mathrm{mg} / \mathrm{dl})$ & 96.9 & 23.8 & 91.6 & 14.7 & 0.02 \\
\hline $\mathrm{HbA} 1 \mathrm{c}(\%)$ & $5 \cdot 6$ & 0.6 & 5.5 & 0.4 & 0.001 \\
\hline HOMA-IR & $2 \cdot 2$ & 2.5 & $1 \cdot 77$ & $1 \cdot 7$ & 0.008 \\
\hline Insulin ( $\mu \mathrm{g} / \mathrm{l})$ & $8 \cdot 15$ & $6 \cdot 2$ & 7.33 & 5.9 & 0.02 \\
\hline Systolic blood pressure $(\mathrm{mmHg})$ & $134 \cdot 4$ & $20 \cdot 4$ & $128 \cdot 7$ & $16 \cdot 9$ & 0.0002 \\
\hline Diastolic blood pressure $(\mathrm{mmHg})$ & 83.8 & $12 \cdot 1$ & 82.1 & 11.1 & 0.08 \\
\hline BMI $\left(\mathrm{kg} / \mathrm{m}^{2}\right)$ & $27 \cdot 4$ & $5 \cdot 2$ & 25.9 & 4.7 & 0.0001 \\
\hline WC $(\mathrm{cm})$ & 91.4 & $14 \cdot 1$ & $88 \cdot 14$ & $13 \cdot 3$ & 0.0028 \\
\hline Total cholesterol (mg/dl) & 208.1 & $42 \cdot 8$ & 201.38 & 39.9 & 0.04 \\
\hline HDL-cholesterol (mg/dl) & 61.8 & 13.5 & 61.78 & $17 \cdot 0$ & 0.87 \\
\hline LDL-cholesterol (mg/dl) & $128 \cdot 7$ & 37.0 & 123.9 & 33.9 & 0.12 \\
\hline TAG $(\mathrm{mg} / \mathrm{dl})$ & $127 \cdot 0$ & 123.5 & 114.5 & 93.2 & 0.08 \\
\hline \multicolumn{6}{|l|}{ Hepatic biomarkers } \\
\hline$\gamma$-GT (mg/l) & 39.65 & 40.4 & 31.5 & $41 \cdot 2$ & 0.0019 \\
\hline AST (mg/l) & $22 \cdot 7$ & 8.14 & $22 \cdot 4$ & 8.9 & 0.55 \\
\hline ALT $(\mathrm{mg} / \mathrm{l})$ & $26 \cdot 2$ & $15 \cdot 3$ & $25 \cdot 1$ & $15 \cdot 4$ & 0.13 \\
\hline
\end{tabular}

FPG, fasting plasma glucose; HbA1c, glycated Hb; HOMA-IR, homoeostasis model assessment of insulin resistance; WC, waist circumference; $\gamma$-GT, $\gamma$-glutamyl-transpeptidase;

AST, aspartate transaminase; ALT, alanine transaminase.

* $P$ values for testing the differences among dietary and cardiometabolic variables across two groups of daily chocolate consumption using analysis of variance.

$\dagger P$ was computed with the Wilcoxon's test.

between increased chocolate consumption and lower levels of ALT, also a marker of inflammation, indirectly confirms previous in vitro results, suggesting that the mechanism through which cocoa may be beneficial to liver cells is more related to its inflammatory effect

Although our findings and other similar cross-sectional studies ${ }^{(41-43)}$ have shown an association between daily chocolate consumption and lower BMI, a more recent prospective study, with 3-year follow-up among postmenopausal American women aged 50-79 years, found a direct association between chocolate-candy consumption and weight gain ${ }^{(44)}$. The divergent findings from these two types of studies could be due to confounding effects of serious chronic diseases ${ }^{(44)}$. Overweight and obese subjects (presented with higher BMI) may tend to decrease their energy-dense food consumption, owing to associated cardiovascular illness or to stay fit. In addition, chocolate intake is likely to be underestimated by those with a higher $\mathrm{BMI}^{(30)}$. In our analyses, we excluded participants who were (at the period of the survey without specifically referring to the number of years before the survey) on a diet. The participants, via a self-reported question, were asked whether they modified their dietary habits to stay in form or because of their awareness of several pathologies (hypertension, lipid disorders, hyperglycaemia or any other reason). This information regarding dieting should help, to some extent, in minimising the potential for reverse causation, and thus avoid a major bias in the observed associations. In our studied sample, chocolate consumption was higher among the younger age group of participants. The relatively healthy profile of chocolate consumers compared with non-consumers may be related to their age, as younger age groups are still healthy and less exposed to age-related pathologies such as diabetes, hypertension and obesity. Adjusting for a wide range of variables including age, sex and socio-economic, lifestyle and dietary factors suggests an independent relationship of these potential confounding factors. These measures may help reduce the potential reverse causality bias.

All glycaemic and hepatic biomarkers of interest were also significantly associated with BMI. After adjusting for BMI in model III, the observed associations were no longer significant (data not shown). In this study, we focused on the examination of the total causal effect. Therefore, it is not only unnecessary but also likely harmful to adjust for a variable (or a proxy of a variable) on a causal path from exposure to disease ${ }^{(26)}$. 
Table 3. Regression coefficients relating chocolate consumption ( $100 \mathrm{~g} / \mathrm{d})$ to glycaemic and hepatic markers, Observation of Cardiovascular Risk Factors in Luxembourg 2007-2008*

(Regression coefficients with their standard errors; $n 1128$ subjects)

\begin{tabular}{|c|c|c|c|c|}
\hline & \multicolumn{4}{|c|}{ Daily chocolate consumption $(100 \mathrm{~g} / \mathrm{d})$} \\
\hline & Covariate sets & $b$ & SE & $P$ \\
\hline \multirow[t]{3}{*}{ FPG (mg/dl) } & Model I† & -0.0049 & 0.0082 & 0.55 \\
\hline & Model II & -0.0028 & 0.0089 & 0.75 \\
\hline & Model III§ & -0.0027 & 0.0089 & 0.76 \\
\hline \multirow[t]{3}{*}{ HbA1c (\%) } & Model I† & -0.002 & 0.0041 & 0.62 \\
\hline & Model II & -0.0062 & 0.0045 & 0.17 \\
\hline & Model III§ & -0.0062 & 0.0045 & 0.17 \\
\hline \multirow[t]{3}{*}{ HOMA-IR } & Model I† & -0.1718 & 0.0523 & 0.001 \\
\hline & Model II & -0.1688 & 0.0572 & 0.003 \\
\hline & Model III§ & -0.1644 & 0.0573 & 0.004 \\
\hline \multirow[t]{3}{*}{ Insulin $(\mu \mathrm{g} / \mathrm{l})$} & Model I† & -0.1633 & 0.0488 & 0.0008 \\
\hline & Model II & -0.1602 & 0.0533 & 0.003 \\
\hline & Model III§ & -0.157 & 0.0533 & 0.003 \\
\hline \multirow[t]{3}{*}{$\gamma$-GT (mg/l) } & Model It & -0.1163 & 0.043 & 0.007 \\
\hline & Model II & -0.1198 & 0.0461 & 0.009 \\
\hline & Model III§ & -0.1199 & 0.0459 & 0.009 \\
\hline \multirow{3}{*}{ AST (mg/l) } & Model It & -0.0264 & 0.0212 & 0.21 \\
\hline & Model II & -0.0394 & 0.0229 & 0.08 \\
\hline & Model III§ & -0.0399 & 0.0228 & 0.08 \\
\hline \multirow[t]{3}{*}{ ALT (mg/l) } & Model I† & -0.0666 & 0.0319 & 0.037 \\
\hline & Model II & -0.0972 & 0.0343 & 0.005 \\
\hline & Model III§ & -0.0983 & 0.0341 & 0.004 \\
\hline
\end{tabular}

FPG, fasting plasma glucose; HbA1c, glycated Hb; HOMA-IR, homoeostasis model assessment of insulin resistance; $\gamma$-GT, $\gamma$-glutamyl-transpeptidase; AST, aspartate transaminase; ALT, alanine transaminase.

* Subjects taking antidiabetic medications ( $n$ 25) were excluded from glycaemic biomarker models (FPG, HbA1c, HOMA-IR and insulin).

† Model I: adjusted for age, sex, education.

‡ Model II: adjusted for age, sex, education, smoking status, physical activity, fruit and vegetable intake, total daily energy intake, alcohol intake.

$\S$ Model III: adjusted for covariates in model II + coffee and tea consumption.

Schisterman et $a l .{ }^{(26)}$ suggest that one is fairly well protected from analytic pitfalls by avoiding control for factors affected by the exposure.

Metabolic benefits from dark chocolate consumption have been demonstrated with regard to BP as well as total cholesterol and LDL-cholesterol levels in short randomised trials ${ }^{(17,18)}$. In addition, chocolate consumption has been linked to lower cardiovascular and all-cause mortality in prospective observational studies ${ }^{(45)}$. The scientific data indicating that cocoa flavanols may make an important contribution to cardiovascular health continues to grow rapidly. Our findings extend favourable associations of chocolate intake with cardiometabolic factors and add evidence for an inverse association between daily chocolate consumption and insulin sensitivity.

The present study has several important strengths. The data were derived from a recent European nationwide populationbased sample. Extensive data on a wide range of potential dietary and lifestyle confounding variables were considered for the multivariable analyses, which have been rarely controlled for in other prospective studies or intervention trials. Furthermore, this is the first study to assess the simultaneous consumption of polyphenol-rich tea and coffee beverages, although residual confounding by additional unknown or unmeasured behavioural or other dietary factors cannot be ruled out.

Multiple components in chocolate, particularly flavonoids, may contribute to the complex interplay between nutrition and health. Cocoa and chocolate contribute to trace-mineral intake, which is necessary for optimum functioning of all biological and vascular systems. In the context of nutrition, this research should be pursued in parallel with further investigation of the potential benefits from chocolate on cognitive and physical health. Our findings add evidence to the growing scientific interest to define nutritional recommendations for dietary polyphenols. Cocoa-based products may offer an extraordinary opportunity to successfully improve compliance to dietary recommendations. Potential applications of this knowledge include recommendations by healthcare professionals to encourage individuals to consume a wide range of phytochemical-rich foods, which can include dark chocolate in moderate amounts ${ }^{(33)}$. It is important to differentiate between the natural product cocoa and the processed product chocolate, which refers to the combination of cocoa, sugar and eventually milk and other ingredients into a solid food product ${ }^{(7)}$. Chocolate is an energy-dense food and individuals must keep energy intake and expenditure in mind when including it in their diet, as any excess may cause an increase in weight. Therefore, physical activity, diet and other lifestyle factors must be carefully balanced to avoid detrimental weight gain over time.

The most predominant limitation of this study was the cross-sectional design. Although the matter of causality cannot be replied, these results may form the basis for generating new hypotheses regarding the relationship between chocolate consumption and insulin resistance and liver enzymes. In addition, despite the intensive efforts to minimise dietary 
reporting inaccuracies through staff training and extensive control procedures ${ }^{(19)}$, self-reported data may be subject to misclassification and recall errors. Different chocolate products yield different amounts of fat, sugar and energy content. Unfortunately, no data were available about the types of chocolate consumed or the amount of flavanols present in finished food products. Likewise, we do not have information about the consumption of cocoa beverages such as milkshakes, hot cocoa, etc., which may confer some drawbacks to our findings.

In conclusion, this study presents some novel findings, supporting a potential beneficial effect of daily chocolate consumption on insulin sensitivity and hepatic biomarkers. Taking the cross-sectional study design into account, further observational prospective research and well-designed randomised-controlled studies are needed to confirm this relationship and to comprehend the role and mechanisms that different types of chocolate may play in insulin resistance and cardiometabolic disorders.

\section{Acknowledgements}

The authors are grateful to Stephen Senn for his valuable comments on the statistical analyses and revision of the manuscript.

A. A. is supported by a grant from the Fond National de Recherche for the DIQUA-LUX project (5870404), Luxembourg (Assessment of Diet Quality of the General Population in Luxembourg and its Association with Cardiometabolic Risk). G. E. C. is supported by a Sidney Sax Research Fellowship (National Health and Medical Research Council, Australia; grant no. APP1054567). The funding sources had no involvement in the study design or in the collection, analysis and interpretation of data.

A. A. was involved in the conception and design of the ORISCAV-LUX survey, coordinated the field data collection, conceived the present research, performed the statistical analyses and drafted the manuscript. N. S. contributed to the statistical analyses and data interpretation. G. E. C., M. F. E. and S. S. contributed to the critical revisions of the intellectual content of the manuscript. All the authors approved the final version of the manuscript.

There are no conflicts of interest to declare.

\section{References}

1. Rosamond WD, Chambless LE, Folsom AR, et al. (1998) Trends in the incidence of myocardial infarction and in mortality due to coronary heart disease, 1987 to 1994 . N Engl J Med 339, 861-867.

2. Steinberger J \& Daniels SR, American Heart Association Atherosclerosis Hypertension, and Obesity in the Young Committee (Council on Cardiovascular Disease in the Young), et al. (2003) Obesity, insulin resistance, diabetes, and cardiovascular risk in children: an American Heart Association scientific statement from the Atherosclerosis, Hypertension, and Obesity in the Young Committee (Council on Cardiovascular Disease in the Young) and the Diabetes Committee (Council on Nutrition, Physical Activity, and Metabolism). Circulation 107, 1448-1453.

3. Ginsberg HN (2000) Insulin resistance and cardiovascular disease. J Clin Invest 106, 453-458.

4. McFarlane SI, Banerii M \& Sowers JR (2001) Insulin resistance and cardiovascular disease. J Clin Endocrinol Metab 86, 713-718.
5. Balkau B \& Eschwege E (1999) Insulin resistance: an independent risk factor for cardiovascular disease? Diabetes Obes Metab 1, Suppl. 1, S23-S31.

6. Ding EL, Hutfless SM, Ding X, et al. (2006) Chocolate and prevention of cardiovascular disease: a systematic review. Nutr Metab (Lond) 3, 2.

7. Corti R, Flammer AJ, Hollenberg NK, et al. (2009) Cocoa and cardiovascular health. Circulation 119, 1433-1441.

8. Lee KW, Kim YJ, Lee HJ, et al. (2003) Cocoa has more phenolic phytochemicals and a higher antioxidant capacity than teas and red wine. J Agric Food Chem 51, 7292-7295.

9. Manach C, Scalbert A, Morand C, et al. (2004) Polyphenols: food sources and bioavailability. Am J Clin Nutr 79, 727-747.

10. Di Castelnuovo A, di Giuseppe R, Iacoviello L, et al. (2012) Consumption of cocoa, tea and coffee and risk of cardiovascular disease. Eur J Intern Med 23, 15-25.

11. Hertog MG, Kromhout D, Aravanis C, et al. (1995) Flavonoid intake and long-term risk of coronary heart disease and cancer in the seven countries study. Arch Intern Med $\mathbf{1 5 5}$, 381-386.

12. Hertog MG, Feskens EJ, Hollman PC, et al. (1993) Dietary antioxidant flavonoids and risk of coronary heart disease: the Zutphen Elderly Study. Lancet 342, 1007-1011.

13. Hartley L, Flowers N, Holmes J, et al. (2013) Green and black tea for the primary prevention of cardiovascular disease. The Cochrane Database of Systematic Reviews 2013, issue 6, CD009934.

14. Keli SO, Hertog MG, Feskens EJ, et al. (1996) Dietary flavonoids, antioxidant vitamins, and incidence of stroke: the Zutphen Study. Arch Intern Med 156, 637-642.

15. Knekt P, Jarvinen R, Reunanen A, et al. (1996) Flavonoid intake and coronary mortality in Finland: a cohort study. BMJ 312, 478-481.

16. Gu Y, Yu S \& Lambert JD (2014) Dietary cocoa ameliorates obesity-related inflammation in high fat-fed mice. Eur J Nutr 53, 149-158

17. Grassi D, Lippi C, Necozione S, et al. (2005) Short-term administration of dark chocolate is followed by a significant increase in insulin sensitivity and a decrease in blood pressure in healthy persons. Am J Clin Nutr 81, 611-614.

18. Grassi D, Desideri G, Necozione S, et al. (2008) Blood pressure is reduced and insulin sensitivity increased in glucose-intolerant, hypertensive subjects after 15 days of consuming high-polyphenol dark chocolate. J Nutr 138, $1671-1676$

19. Alkerwi A, Sauvageot N, Donneau AF, et al. (2010) First nationwide survey on cardiovascular risk factors in GrandDuchy of Luxembourg (ORISCAV-LUX). BMC Public Health 10, 468.

20. Alkerwi A, Sauvageot N, Couffignal S, et al. (2010) Comparison of participants and non-participants to the ORISCAV-LUX population-based study on cardiovascular risk factors in Luxembourg. BMC Med Res Methodol 10, 80.

21. Sauvageot N, Alkerwi A, Albert A, et al. (2013) Validation of the food frequency questionnaire used to assess the association between dietary habits and cardiovascular risk factors in the NESCAV study. J Nutr Food Sci 3, 208.

22. Sauvageot N, Alkerwi A, Albert A, et al. (2013) Use of food frequency questionnaire to assess relationships between dietary habits and cardiovascular risk factors in NESCAV study: validation with biomarkers. Nutr J 12, 143.

23. Bonora E, Kiechl S, Willeit J, et al. (2007) Insulin resistance as estimated by homeostasis model assessment predicts incident symptomatic cardiovascular disease in Caucasian subjects from the general population: the Bruneck study. Diabetes care 30, 318-324. 
24. Alkerwi A, Sauvageot N, Nau A, et al. (2012) Population compliance with national dietary recommendations and its determinants: findings from the ORISCAV-LUX study. Br J Nutr 108, 2083-2092.

25. Alkerwi A, Donneau AF, Sauvageot N, et al. (2012) Dietary, behavioural and socio-economic determinants of the metabolic syndrome among adults in Luxembourg: findings from the ORISCAV-LUX study. Public Health Nutr 15, 849-859.

26. Schisterman EF, Cole SR \& Platt RW (2009) Overadjustment bias and unnecessary adjustment in epidemiologic studies. Epidemiology 20, 488-495.

27. Mancia G, De Backer G, Dominiczak A, et al. (2007) 2007 Guidelines for the Management of Arterial Hypertension: The Task Force for the Management of Arterial Hypertension of the European Society of Hypertension (ESH) and of the European Society of Cardiology (ESC). J Hypertens 25, 1105-1187.

28. Ryden L, Standl E, Bartnik M, et al. (2007) Guidelines on diabetes, pre-diabetes, and cardiovascular diseases: executive summary. The Task Force on Diabetes and Cardiovascular Diseases of the European Society of Cardiology (ESC) and of the European Association for the Study of Diabetes (EASD). Eur Heart J 28, 88-136.

29. De Backer G, Ambrosioni E, Borch-Johnsen K, et al. (2003) European guidelines on cardiovascular disease prevention in clinical practice: third joint task force of European and other societies on cardiovascular disease prevention in clinical practice (constituted by representatives of eight societies and by invited experts). Eur J Cardiovasc Prev Rehabil 10, S1-S10.

30. Buitrago-Lopez A, Sanderson J, Johnson L, et al. (2011) Chocolate consumption and cardiometabolic disorders: systematic review and meta-analysis. BMJ 343, d4488.

31. Hooper L, Kroon PA, Rimm EB, et al. (2008) Flavonoids, flavonoid-rich foods, and cardiovascular risk: a meta-analysis of randomized controlled trials. Am J Clin Nutr 88, 38-50.

32. Murphy MM, Barraj LM, Bi X, et al. (2013) Body weight status and cardiovascular risk factors in adults by frequency of candy consumption. Nutr J 12, 53.

33. Steinberg FM, Bearden MM \& Keen CL (2003) Cocoa and chocolate flavonoids: implications for cardiovascular health. J Am Diet Assoc 103, 215-223.
34. Brand-Miller J, Holt SH, de Jong V, et al. (2003) Cocoa powder increases postprandial insulinemia in lean young adults. J Nutr 133, 3149-3152.

35. Grassi D, Necozione S, Lippi C, et al. (2005) Cocoa reduces blood pressure and insulin resistance and improves endothelium-dependent vasodilation in hypertensives. Hypertension 46, 398-405.

36. Carrieri MP, Lions C, Sogni P, et al. (2014) Association between elevated coffee consumption and daily chocolate intake with normal liver enzymes in HIV-HCV infected individuals: results from the ANRS CO13 HEPAVIH cohort study. J Hepatol 60, 46-53.

37. Selmi C, Cocchi CA, Lanfredini M, et al. (2008) Chocolate at heart: the anti-inflammatory impact of cocoa flavanols. $\mathrm{Mol}$ Nutr Food Res 52, 1340-1348.

38. Katz DL, Doughty K \& Ali A (2011) Cocoa and chocolate in human health and disease. Antioxid Redox Signal 15, 2779-2811.

39. Zeng G, Nystrom FH, Ravichandran LV, et al. (2000) Roles for insulin receptor, PI3-kinase, and Akt in insulin-signaling pathways related to production of nitric oxide in human vascular endothelial cells. Circulation 101, 1539-1545.

40. Potenza MA, Marasciulo FL, Tarquinio M, et al. (2007) EGCG, a green tea polyphenol, improves endothelial function and insulin sensitivity, reduces blood pressure, and protects against myocardial I/R injury in SHR. Am J Physiol Endocrinol Metab 292, E1378-E1387.

41. Golomb BA, Koperski S \& White HL (2012) Association between more frequent chocolate consumption and lower body mass index. Arch Intern Med 172, 519-521.

42. Greenberg JA \& Buijsse B (2013) Habitual chocolate consumption may increase body weight in a doseresponse manner. PLOS ONE 8, e70271.

43. Cuenca-Garcia M, Ruiz JR, Ortega FB, et al. (2014) Association between chocolate consumption and fatness in European adolescents. Nutrition 30, 236-239.

44. Greenberg JA, Manson JE, Buijsse B, et al. (2015) Chocolatecandy consumption and 3-year weight gain among postmenopausal U.S. women. Obesity (Silver Spring) 23, 677-683.

45. Buijsse B, Feskens EJ, Kok FJ, et al. (2006) Cocoa intake, blood pressure, and cardiovascular mortality: the Zutphen Elderly Study. Arch Intern Med 166, 411-417. 